\title{
Cutaneous Flaps for Closing Skin Defects in Dogs
}

\author{
Florin BETEG*, Aurel MUSTE \\ University of Agricultural Sciences and Veterinary Medicine, Faculty of Veterinary Medicine, Surgery \\ Clinic, Calea Mănăștur 3-5, 400372, Cluj Napoca, Romania \\ *corresponding author: florin.beteg@usamvcluj.ro
}

Bulletin UASVM Veterinary Medicine 73(2) / 2016,

Print ISSN 1843-5270; Electronic ISSN 1843-5378

DOI:10.15835/buasvmcn-vm: 12262

\begin{abstract}
Cutaneous flaps are used for closing wounds caused by traumatic accidents, oncological surgery (tumor removal), and burns (thermal, chemical, radiations). Skin grafting has the advantages of requiring just only one surgery for closing the defects once the wound bed is adequately prepared.

The objective of the study was to describe and asses the efficiency of local cutaneous flaps for closing skin defects in dogs.

Seven dogs underwent reconstruction of soft tissue wounds resulting from traumatic lesion or after large tumors removal. Skin defects were located on the trunk and limbs. Cutaneous local flaps (advancement and rotational) were created by surgical preparations and mobilization of full thickness skin fold to enable closure of adjacent defects. After wound debridement or tumoral removal a very careful atraumatic and aseptical preparation of the flaps were performed to preserve vascularization for adequate blood supply.

Cutaneous local flaps proved effective for closing large defects in all dogs. Partial marginal necrosis of a portion of the flap occurred in one dog because of procedure and technique errors, but the concurrent remanent defects were adequate to primary closure. The wounds ultimately healed, without major complications.

The skin local flaps (advancement and rotational) are a versatile technique that could be used in a variety of locations, depending on skin defects shape and localization. The clinical results are comparable with those reported for advanced reconstructive procedure.
\end{abstract}

Keywords: cutaneous, defect, dog, flaps, skin.

\section{INTRODUCTION}

Local (subdermal) cutaneous flaps are a way of reconstructing large open wounds by using adjacent or regional skin that is advanced or rotated to allow the edge apposition and closure. A skin flap (pedicle flap) is a partially detached segment of skin and subcutaneous tissue used to cover the defects (Fowler, 1999). Local or regional blood vessels supplying these flaps do not require a bed of granulation tissue for their blood supply and nutrition. Skin flaps provide immediate coverage of the defects and prevent the prolonged healing, excessive scar tissue formation, and contracture associated with secondary healing intention (Dernell, 2006). They can be classified in various ways depending on the location, blood supply and tissue formation. Based on these criteria they are classified as local subdermal plexus flaps and axial pattern flaps. Local subdermal plexus flaps are those designed with skin adjacent to the wound and are based on direct cutaneous artery (Pavletic, 1990). Axial pattern flaps are those that are developed using a major direct cutaneous artery as primary blood supply. Local subdermal flaps are classified according to their technique of transfer: advancement flaps which advance in a forward direction and rotational flaps which rotate on pivot into position (Hedlund, 2006).

\section{MATERIALS AND METHODS}

The observations in this study were performed on a total of seven dogs of various breeds, ages and gender which were presented at the Surgical Clinic for diagnosis and treatment. Dogs in the 


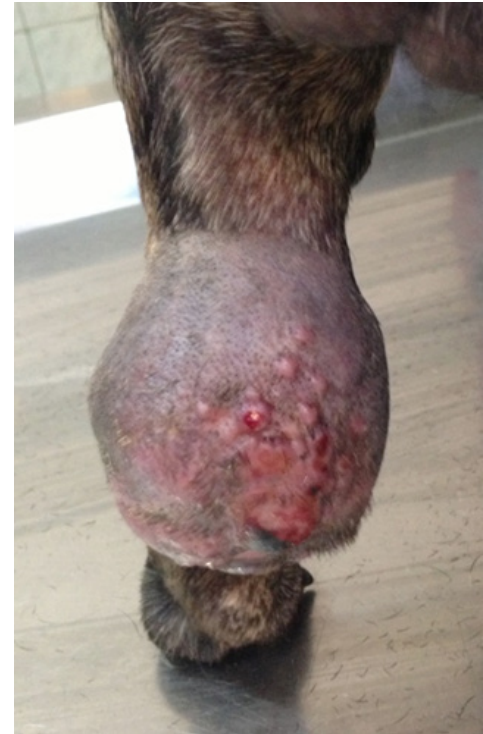

Fig. 1. Tumor appearance prior to removal

study had skin defects (acute or chronic) after traumatic injuries or large wounds resulted after large tumor ablation (Figure 1).

Skin defects were located on the trunk and limbs. The patients were carefully evaluated to ensure the chance of flap survival. The dogs underwent general inhalatory anesthesia with Isuflurane after premedication with Domitor and Butomidor and induction with Propofol. A large area was clipped and aseptically prepared for surgery. A skin marker was used to outline the anticipated skin incisions (Figure 2).

Cutaneous defects resulted after tumor removal and wound debridement were prepared by removing all modified tissue to be sure that the exposed bed is free of contamination and infection. Cutaneous local flaps (advancement and rotational) were created by surgical preparations and mobilization the full tickness skin fold to enabling closure of adjacent defects. Before creating the flap, the skin adjacent to the wounds was manipulated to asses elasticity and lines of tension and to identify the direction in which the skin will easily move. After wound debridment or tumoral removal, a very careful atraumatic and aseptical preparation of the flaps were performed to preserve vascularization for adequate blood supply, not to damage the subdermal plexus.

To provide sufficient mobility, the length of flap was as long as the length of the wound to be closed. Flaps were designated with the base wider than the overall width of the flap and the length of

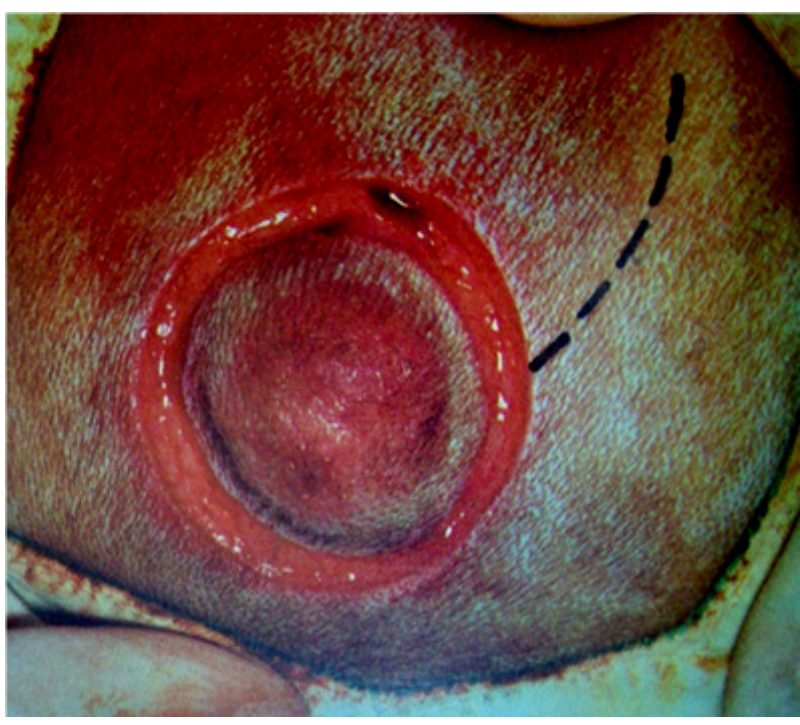

Fig.2. Skin incision and flap outline

the defect to be closed. Closures of the flaps were performed in two layers with absorbable sutures in the subcutaneous tissue and nonabsorbable sutures in the skin (Figure 3).

The wounds were covered by light protective bandagingfor 2 days. Antibiotics were administered postoperatively as Cephalexin (Solvasol) at 20 $\mathrm{mg} / \mathrm{kg}$ once daily continued for 3 days, depending on the primary disease process and analgesia was supplied by carprofen (Rimadyl) given at $2.2 \mathrm{mg} /$ $\mathrm{kg}$ twice daily for 5 days.

\section{RESULTS AND DISCUSSION}

Postoperative all dogs recovered well and were monitored in the first week daily for flaps survival and aseptical and septical complication. The flaps exhibited some postoperative congestion and edema in the first three days postop. Areas of necrosis approximately $10 \mathrm{~mm} \times 30 \mathrm{~mm}$ developed in one advancement monopedicle flap. This was excised and the resulting defect closed without difficulty in a subsequent procedure undertaken a week after the first one. Thereafter healing progressed well with sutures removed after 10 days. Other important potential reasons for flap necrosis include kinking, seroma, haematoma formation and infection (Vasconcellos, 2010). Careful preoperative planning and atraumatic surgical technique are required to minimize these problems (Hunt, 2001; Nevill, 2010).

Maintaining a body wall attachment may lead to better flap survival. Depending on width of the 


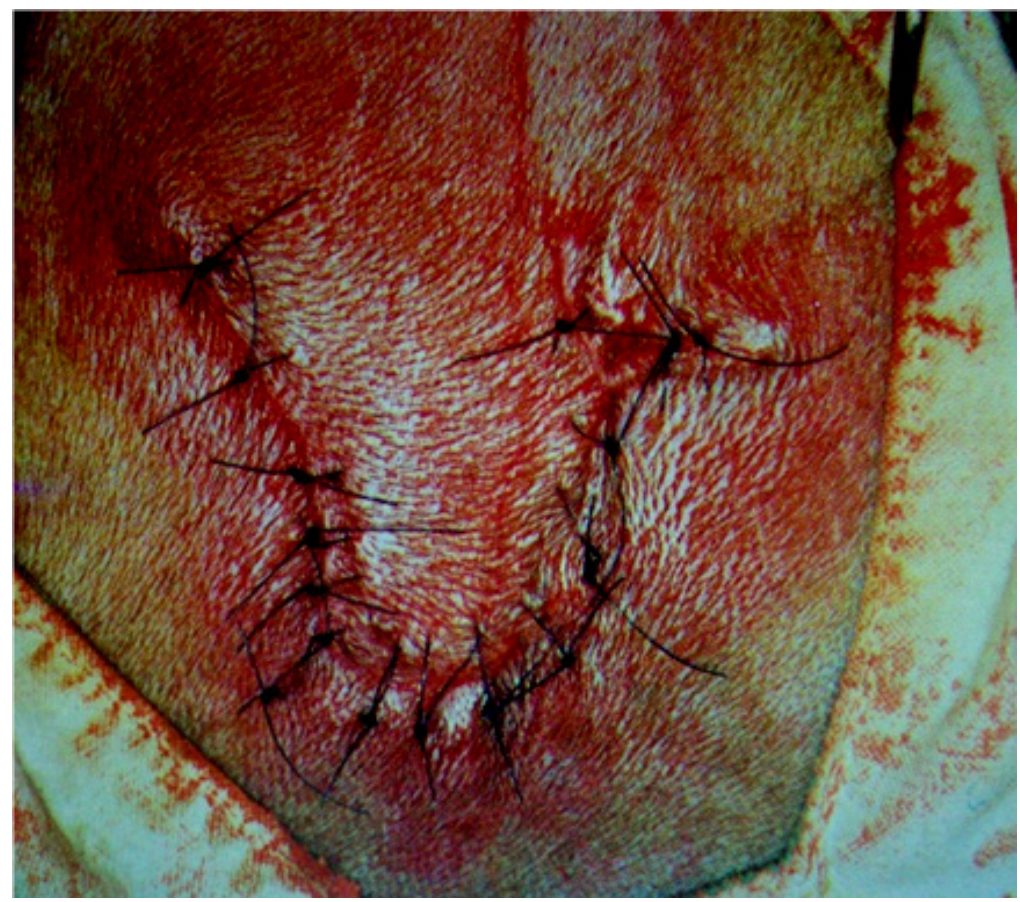

Fig.3. Skin flap suture

base where the base is positioned, it is possible to cover areas larger or more restricted. The amount of skin available to harvest depends on the particular conformation of the patient.

Postoperative care in all skin flap procedures should include adequate antibiotherapy, analgesia because these procedures are very painful. Postoperative cage confinement will also help limit undesirable movement and tension on the skin flaps. Drains are an important consideration as they help to limit dead space formation and fluid accumulation (Nevill, 2010).

\section{CONCLUSSIONS}

The advancement pedicle flaps are suitable for the skin defect located on the extremities where tissue is inadequate to rotate the flap and a conventional elliptical excision could provide no means for closure.

The rotational flaps are ideal for large skin defects located on the trunk; a wide base of the flap must be maintained to provide a generous supply of blood to the graft.

\section{REFERENCES}

1. Dernell WS (2006). Initial wound management. Veterinary Clinics of North America, Small Animal Practice 713-73.

2. Fowler D (1999). Tension relieving techniques and local skin flaps. In Fowler D, Williams J M (eds) BSAVA manual of canine and feline wound management and reconstruction, Cheltenham: 59-68.

3. Hedlund CS (2006). Large trunk wounds. Veterinary Clinics of North America, Small Animal Practice 36: 846872.

4. Hunt GB, Tisdall PLC, Liptak JM, Beck JA, Swinney GR, Malik R (2001). Skin-fold advancement flaps for closing large proximal limb and trunk defects in dogs and cats. Veterinary Surgery 30: 440-448.

5. Nevill BG (2010). Bilateral axillary skin folds flaps used for dorsal thoracic skin wound closure in a dog. Journal of the South African Veterinary Association 81(1): 58-61.

6. Pavletic M (1990). Skin flaps in reconstructive surgery. Veterinary Clinics of North America Small Animal Practice: 80-103.

7. Vasconcellos CH, Matera JM, Dagli MLZ (2005). Clinical evaluation of random skin flaps based on the subdermal plexus secured with sutures or sutures and cyanoacrylate adhesive for reconstructive surgery in dogs. Veterinary Surgery 34: 59-63. 